\title{
Supplementary Information: Pattern Development and Control of Strained Solitons in Graphene Bilayers
}

Shizhe Feng and Zhiping $\mathrm{Xu}^{*}$

Applied Mechanics Laboratory, Department of Engineering Mechanics, Tsinghua University, Bei-

jing 100084, China

*email: xuzp@tsinghua.edu.cn

This Supplementary Information Material includes

Supplementary Notes S1-S5. Detailed description of simulation methods and theoretical analysis,

including Simulation Methods, Deformable Tension-Shear Model, Order Parameters, Two-Chain

Frenkel-Kontorova Model, Dislocation Theory for Bilayer Graphene.

\section{Supplementary Figures S1-S13.}




\section{NOTE 1 SIMULATION METHODS}

Two graphene sheets with an identical length $L$ ranging from 10 to $1000 \mathrm{~nm}$ were constructed in perfect $\mathrm{AB}$ stacking. A ribbon-like geometry is used, where a periodic boundary condition (PBC) is applied along the width direction, with $w \approx 2 \mathrm{~nm}$. Open boundaries are enforced for the other two directions. Molecular dynamics (MD) simulations were performed using the largescale atomic/molecular massively parallel simulator (LAMMPS) $)^{1}$. The second-generation reactive empirical bond order (REBO) potential ${ }^{2}$ is used for monolayers, and the interlayer interaction is modeled by the registry-dependent Kolmogorov-Crespi (K-C) potential with a taper function ${ }^{3 / 4}$. To simulate the tensile tests, stretch and energy minimization are performed step by step. Specifically, two opposite ends (two rows of hexagons for each) of the graphene bilayer are pulled with a step size of $0.1 \AA$ (Fig. 1), followed by structural relaxation using the conjugate gradient (CG) algorithm with a force tolerance of $10^{-6} \mathrm{eV} / \AA^{5}$. To model substrate adhesion, the out-of-plane displacement of atoms in the bottom layer is fixed. To map the simulated systems into a one-dimensional (1D) problem for theoretical analysis, the size of simulation box in the transverse direction is fixed if not specified. To turn on the Poisson's effect, lattice deformation in that direction is allowed. The atomic stress is calculated following the Virial definition, ${ }^{[6}$ with the thickness of graphene defined as $t=0.335 \mathrm{~nm}$. For finite-temperature simulations, a Berendsen thermostat with the timestep of 0.001 picoseconds is applied. To explore the reversibility of soliton structures, the stretch force is released first. The bilayer system is then heated and relaxed at $10 \mathrm{~K}$ to avoid trapping events at local minima of the potential energy landscape. 


\section{NOTE 2 DEFORMABLE TENSION-SHEAR MODEL}

In the deformable tension-shear (DTS) model for a graphene bilayer (Fig. S12), the elastic sheets with tensile stiffness $D=Y t$ are interacting through a continuum with interlayer shear modulus $G_{\mathrm{I}}$, where $Y$ and $t$ are the Young's modulus and thickness of graphene. Our discussion here differs from that in Ref. ${ }^{[7}$ by the fact that a bilayer model is analyzed for the proposed experimental setup in Fig. 1 instead of the layer-by-layer assembly for graphene films ${ }^{7}$. Under a tensile force $F_{0}$ (per unit length along the width), we denote the in-plane displacement in each layer as $u_{1}(x)$ and $u_{2}(x)$, where $x$ is the coordinate along the loading direction. The equation of mechanical equilibrium is $D \frac{\partial^{2} u(x)}{\partial x^{2}}=G_{\mathrm{I}} \gamma(x)$, where $\gamma(x)=\left[u_{2}(x)-u_{1}(x)\right] / h$ is the shear strain calculated from the relative displacement between these two platelets. $h=0.335 \mathrm{~nm}$ is the interlayer distance.

The model can be solved with force boundary conditions $D \frac{\partial u_{1}(L)}{\partial x}=D \frac{\partial u_{2}(0)}{\partial x}=0$ at the free ends, and $D \frac{\partial u_{1}(0)}{\partial x}=D \frac{\partial u_{2}(L)}{\partial x}=F_{0}$ at the ends with force applied, yielding the distribution of interior force and shear strain as

$$
\begin{gathered}
F(x)=\frac{F_{0}}{2}\left[1-\cosh \left(\frac{x}{l_{0}}\right)+\frac{1+c}{s} \sinh \left(\frac{x}{l_{0}}\right)\right], \\
\gamma(x)=\frac{F_{0} l_{0}}{D h}\left[\frac{1+c}{s} \cosh \left(\frac{x}{l_{0}}\right)-\sinh \left(\frac{x}{l_{0}}\right)\right],
\end{gathered}
$$

where $s=\sinh \left(L / l_{0}\right), c=\cosh \left(L / l_{0}\right) . l_{0}=\left(D h / 2 G_{\mathrm{I}}\right)^{1 / 2}$ is the characteristic length of contact required for effective load transfer between adjacent platelets through shear. 
The effective Young's modulus is defined as $Y_{\text {eff }}=\sigma / \varepsilon$, where the effective stress and strain are $\sigma=F_{0} / h$ and $\varepsilon=\left[u_{1}(L)-u_{2}(0)\right] / L$, that is

$$
Y_{\mathrm{eff}}=\frac{D}{h} /\left(\frac{1}{2}+\frac{1+c}{s} \frac{l_{0}}{L}\right) .
$$

The mechanism of failure is determined by the in-plane elasticity of platelets and the interaction between them through shear. The maximum shear strain defined in the interlayer continuum is located at $x=0$ and $L$ according to Eq. S2, Beyond a critical shear strain $\gamma_{\mathrm{cr}}$, the representative volume element (RVE) fails by activating sliding between the platelets, and the effective tensile strength is

$$
\sigma_{\mathrm{s}}=\frac{F_{0}}{h}=\frac{s \gamma_{\mathrm{cr}} D}{(1+c) l_{0}} .
$$

From MD simulations of graphene ${ }^{7}$, the model parameters are determined as $D=Y t=295 \mathrm{~N} / \mathrm{m}$, $h=t=0.335 \mathrm{~nm}, G_{\mathrm{I}}=2.548 \mathrm{GPa}, \gamma_{\mathrm{cr}}=0.144$, and $l_{0}=4.4 \mathrm{~nm}$. The Poisson's ratio is $\nu=0.39$. As the size of overlap between two bilayer $L$ is below $l_{\mathrm{c}}=3 l_{0}=13 \mathrm{~nm}$, the bilayer structure would fail by sliding as interfacial load transfer into lattice deformation of the monolayers is not sufficient. The monolayers can be considered as rigid platelets. DTS predictions align well with the simulation results as the load transfer between monolayers and their elastic deformation are significant $\left(L>3 l_{0}\right)$, while solitons are not nucleated and the continuum assumption of interlayer interaction remains valid (Fig. 1). For $L=300 \mathrm{~nm}$, we have $D=573 \mathrm{~N} / \mathrm{m}$ and $\sigma_{\mathrm{s}} h=3.23 \mathrm{~N} / \mathrm{m}$. 
However, the peak stress after slip activation cannot be calculated using this model. 


\section{NOTE 3 ORDER PARAMETERS}

Interlayer Registry Measures. The registry index (RI) was proposed to quantify the commensurability between $2 \mathrm{D}$ crystals $^{8}$. The value of RI is computed through the overlap area between carbon atoms in different graphene layers, where each carbon atom is considered as a circle with radius $r=a / 2 . a=0.142 \mathrm{~nm}$ is the length of $\mathrm{C}-\mathrm{C}$ bonds in graphene. To measure local slip between 2D crystalline lattices, we define a local registry index (LRI) by spatial averaging over the pairs of $\mathrm{A}, \mathrm{B}$ subsites. In Bernal ( $\mathrm{AB}$ or $\mathrm{BA}$ ) stacking, neighboring RIs for the $\mathrm{C}$ atoms on the subsites are 1 and 0 and the value of LRI is 0.5 , while for AA stacking, the LRI is 1.0.

Atomic Slip Parameters. An atomic slip vector is defined to measure the in-plane displacement of carbon atoms during deformation. For the graphene bilayer, coordinates of carbon atoms in the top layer are projected to local coordinate systems defined by the nearest triplet in the bottom layer in the same subsites (A or B). While atomic slip occurs, the projected position of the $i$-th atom in the top layer is $\mathbf{x}_{\|}^{i}=\sum_{j} k_{j}^{i} \mathbf{x}_{j}^{i}$, where $\mathbf{x}_{j}^{i}$ are the current positions of the reference atom triplet, and $k_{j}^{i}$ is the projection factor. The coordinates can be mapped into a unstrained lattice as $\mathbf{x}_{\|}^{i \prime}=\sum_{j} k_{j}^{i} \mathbf{x}_{0 j}^{i}$.

The atomic slip length (SL) is defined as $\frac{\left|\mathbf{x}_{\|}^{i /}-\mathbf{x}_{\|, 0}^{i}\right|}{a}$, where $\mathbf{x}_{\|, 0}^{i}$ is the original projected position of atom $i$. The value of SL is 0 or 1 for lattices in Bernal stacking. 


\section{NOTE 4 FRENKEL-KONTOROVA TWO-CHAIN MODEL}

Model Description. The soliton behaviors could be effectively captured in a two-chain FrenkelKontorova (FK) model $\mathrm{l}^{910}$, where each graphene layer is treated as a chain of equally-spaced beads that are connected by springs with length $l$ and stiffness $k$. The present model differs from that in Refs .910 by the symmetry of loading, that is, the two graphene layers are pulled at different ends as illustrated in Fig. 1. Considering the in-plane elasticity of tensile and shear, the effective stiffness is $k=Y t \cos ^{2} \phi+G t \sin ^{2} \phi$, where $t$ is the thickness of graphene monolayers, $Y$ and $G=Y / 2(1+\nu)$ are the Young's modulus and intralayer shear modulus of graphene, $v$ is the Poisson's ratio. For our simulation setup, the parameters are $l=a=0.142 \mathrm{~nm}$ (the distance between Bernal configurations along the AB-SP-BA path in MEP, Fig. S1), $Y t=295$ N/m or 18.6 $\mathrm{eV} / \AA^{2}\left(249 \mathrm{~N} / \mathrm{m}\right.$ or $\left.15.6 \mathrm{eV} / \AA^{2}\right)$ for stretching along the armchair (zigzag) directions, respectively. The value of $\nu$ is 0 or 0.39 for the constrained or unconstrained simulations.

We define the relative displacement between neighboring beads in the top and bottom monolayers as $u_{n}$ (Fig. S13, which is $\frac{\mathrm{d} u}{\mathrm{~d} x}$ in the continuum limit. For simplification, a $\mathcal{C}_{2}$ symmetry of the bilayer structure is assumed (Fig. 1). The change in potential energy of interlayer interaction compared with that in Bernal stacking (Fig. S1) can be approximated by a cosine function as

$$
V(u)=V_{\mathrm{SP}}\left[2 \cos \left(\frac{2 \pi}{3} u+\frac{2 \pi}{3}\right)+1\right]^{2}
$$

where $u$ is defined in unit of $l$, which is defined as the $\mathrm{C}-\mathrm{C}$ bond length $a$ in this work, following 
the minimum energy path (MEP) AB-SP-BA in Fig. S1a.

In our setup (Fig. 1), the formation energy of a soliton spanning over $n=-N / 2, \cdots, N / 2$ (from $\mathrm{AB}$ to BA, Fig. S1 1 ) is

$\Delta U=\sum_{-\frac{N}{2}}^{\frac{N}{2}}\left\{\frac{k l^{2}}{2}\left[\left(\lambda_{n+1}+\frac{1}{2} u_{n+1}\right)-\left(\lambda_{n}+\frac{1}{2} u_{n}\right)\right]^{2}+\frac{k l^{2}}{2}\left[\left(\lambda_{n+1}-\frac{1}{2} u_{n+1}\right)-\left(\lambda_{n}-\frac{1}{2} u_{n}\right)\right]^{2}+V(u)\right\}-U_{0}$

where $\lambda_{n} l$ is the displacement of a bead from its equilibrium position, and $U_{0}$ is the energy of the bilayer structure in $\mathrm{AB}$ stacking, that is

$$
U_{0}=\sum_{-\frac{N}{2}}^{\frac{N}{2}}\left[k l^{2}\left(\lambda_{n+1}-\lambda_{n}\right)^{2}\right]
$$

The boundary conditions are $u_{-\frac{N}{2}}=0, u_{\frac{N}{2}}=+1$ for kinks or $u_{\frac{N}{2}}=-1$ for antikinks. The formation energy is then calculated as

$$
\Delta U=U-U_{0}=\sum_{-\frac{N}{2}}^{\frac{N}{2}}\left[\frac{k l^{2}}{4}\left(u_{n+1}-u_{n}\right)^{2}+V(u)\right]
$$

With $N \gg 1$, one approaches the continuum limit. For an isolated single soliton, we can rewrite 
Eq. $\$ 8$ as

$$
\Delta U=\int_{-\infty}^{\infty}\left[\frac{k l^{2}}{4}\left(\frac{\mathrm{d} u}{\mathrm{~d} x}\right)^{2}+V(u)\right] \mathrm{d} x
$$

Applying the variational principle to $\Delta U$ with respect to $u$ yields the Euler-Lagrange equation

$$
\frac{k l^{2}}{4}\left(\frac{\mathrm{d} u}{\mathrm{~d} x}\right)^{2}=V(u)
$$

Critical Strain. In the continuum model (Eq. S9), the in-plane elastic energy density due to elongation is $2 \cdot \frac{1}{2} k \varepsilon^{2}$. Consider the interlayer potential energy surface (PES) as shown in Fig. S1 (a), the critical strain for soliton nucleation by slipping through the MEP can be determined from

$$
k \varepsilon_{\mathrm{cr}}^{2}=V_{\mathrm{SP}}+\frac{1}{2} G \gamma_{x y}^{2}\left(\phi_{\mathrm{s}}\right)
$$

where $V_{\mathrm{SP}}$ is the interlayer potential energy barrier and $\phi_{\mathrm{s}}$ is the value of $\phi$ for a specific soliton. The intralayer shear strain is $\gamma_{x y}=\frac{a \tan \phi}{\lambda_{\mathrm{s}}(\phi)}$, where $\lambda_{\mathrm{s}}$ is the soliton width. For interlayer sliding along the armchair direction, there are a tensile soliton $\left(\phi=0^{\circ}\right)$ and a mixed tension-shear soliton $\left(\phi= \pm 60^{\circ}, \pm 120^{\circ}\right)$. The critical stain values of $\varepsilon_{\mathrm{cr}}$ predicted are thus $\sqrt{\frac{V_{\mathrm{SP}}}{k}}=5.8 \times 10^{-3}$ and $\sqrt{\frac{V_{\mathrm{SP}}+\frac{1}{2} G \gamma_{x y}^{2}}{k}}=1.03 \times 10^{-2}$, respectively. Similarly, along the zigzag direction $\left(\phi= \pm 30^{\circ}, \pm 150^{\circ}\right)$, the value is $\sqrt{\frac{V_{\mathrm{SP}}+\frac{1}{2} G \gamma_{x y}^{2}}{k}}=6.3 \times 10^{-3}$. These results are consistent with the simulation results (Figs. S8] and S9].

Soliton Structures. The kink (+) and antikink (-) solutions for Eq. S10 are 


$$
u_{ \pm}=\frac{1}{2} \pm \frac{3}{\pi} \arctan \left[\frac{1}{\sqrt{3}} \tanh \left(2 \pi x \sqrt{\frac{V_{\mathrm{SP}}}{3 k l^{2}}}\right)\right]
$$

and the width of a soliton is

$$
\lambda_{\mathrm{s}} \simeq\left|\frac{\mathrm{d} u}{\mathrm{~d} x}\right|_{x=0}^{-1}=\frac{l}{2} \sqrt{\frac{k}{V_{\mathrm{SP}}}}
$$

Soliton Patterns. For a chain of kinks or antikinks as identified in our simulation results (Fig. 3), nonlinear periodic solutions of Eq. S10 can be obtained through the Jacobi elliptic function. Using a simplified form for the interlayer potential as $V(u)=\frac{V_{\mathrm{SP}}}{2}\left[1-\cos \left(\pi\left(u-u_{0}\right)\right)\right]$, where $u_{0}$ is the slip length at the boundaries $\left(x_{0}=0\right.$ or $\left.L\right)$. The elastic energy required to create a soliton can be determined by Eq. S9 using the soliton profile

$$
u(x)=\frac{1}{\pi} \arcsin \left[\operatorname{sn}\left(b\left(x-x_{0}\right), m\right)\right]+u_{0}
$$

where $m=\sqrt{\frac{4 \pi^{2} V_{\mathrm{SP}}}{k l^{2} \Delta U}}, b= \pm \sqrt{\frac{4 \pi^{2} \Delta U}{k l^{2}}}$.

From this two-chain model, we can predict the width of solitons $\lambda_{\mathrm{s}}$, their separation $R=2 \mathbf{K}(m) / b$, and the number of soliton $n_{\mathrm{s}}=L / R$, where $\mathbf{K}$ is the complete elliptic integral of the first kind. As $U$ increases with the loading amplitude $\delta, R$ decreases, and the line density of solitons increases (Figs. 4, S8 $\mathrm{S} 11$. Using $\Delta U=2 \cdot \frac{1}{2} k\left(\varepsilon-\varepsilon_{\mathrm{cr}}\right)^{2}$, we can predict a scaling relation $R \sim\left(\varepsilon-\varepsilon_{\mathrm{cr}}\right)^{1.05}$ 
between $R$ and strain in the monolayers, $\varepsilon$. Simulation results for graphene bilayers stretched along the armchair direction can be well fitted by this relation (Fig. S7 $\mathrm{g}$ ). The results of $u(x)$ including both kink and antikink structures with varying $m$ in the model could match simulation results at different values of $\delta\left(u_{0}=0.5\right.$, Fig. $\left.\mathbf{S 7}\right)$. 


\section{NOTE 5 DISLOCATION THEORY FOR BILAYER GRAPHENE}

The kink and antikink structures in bilayer graphene can be analyzed using the dislocation theory $11 \mid 12$. Slip or glide in the $\left(0,0,0, \frac{1}{2}\right)$ plane can be activated by shear as indicated in Fig. S6a. For stretch along the armchair or zigzag direction, the paths of slip $(A B \rightarrow A B$, a full edge dislocation) are composed of two partial dislocations. The stability of dislocations during the loading process is governed by the Frank's rule of dislocation reaction (Fig. S6b), which suggests that, as the elastic energy of a dislocation scales as $\mathbf{b}^{2}$, a reaction is feasible if $\mathbf{b}_{1}^{2}+\mathbf{b}_{2}^{2}>\mathbf{b}_{3}^{2}=\left(\mathbf{b}_{1}+\mathbf{b}_{2}\right)^{2}$ or $\Delta \phi>\frac{\pi}{2}$.

For stretching along the armchair direction $([1 \overline{1} 00])$, we have $\mathbf{b}=a_{0} / 3[\overline{2} 110] \rightarrow a_{0} / 3[1 \overline{1} 00]+$ $a_{0} / 3[0 \overline{1} 10]$ or $\mathbf{b}=a_{0} / 3[2 \overline{11} 0] \rightarrow a_{0} / 3[1 \overline{1} 00]+a_{0} / 3[10 \overline{1} 0]$. Frank's rule suggests that the $0^{\circ}$ solitons annihilate (Fig. S6c), while the $\pm 60^{\circ}$ ones coexist (Fig. S6d). Including the Poisson's effect allows the annihilation of the $\pm 60^{\circ}$ solitons that are located at the center (Fig. S6e). For stretch along the zigzag direction $([\overline{11} 20])$, we have $\mathbf{b}=a_{0} / 3[\overline{11} 20] \rightarrow a_{0} / 3[\overline{1} 010]+a_{0} / 3[0 \overline{1} 10]$. Frank's rule suggests that the $-30^{\circ}$ solitons annihilate (Fig. S6f). Here b is the Burgers vector, $|\mathbf{b}|=a_{0}$, and $a_{0}=\sqrt{3} a$ is the lattice constant of graphene. 


\section{SUPPLEMENTARY FIGURES S1-S13.}

S1. Potential energy surfaces (PES) and kink/antikink structures.

S2. Stress and interlayer registry of bilayer graphene under stretch.

S3. Mechanical responses and PES of bilayer graphene with a twisted angle.

S4. Mechanical responses and atomic slip in bilayer graphene under stretch.

S5. Stress distribution in the bottom layer for bilayer graphene.

S6. Evolution of kink and anitikink structures.

S7. Slip length (SL) distribution in bilayer graphene.

S8. Mechanical responses and atomic slip of bilayer graphene stretched along the armchair direction.

S9. Mechanical responses of bilayer graphene stretched along the zigzag direction.

S10. Mechanical responses and atomic slip of bilayer graphene with the bottom layer constrained.

S11. Mechanical responses and atomic slip of bilayer graphene with the Poisson's effect.

S12. The deformable tensileshear (DTS) model of bilayer graphene.

S13. The two-chain Frenkel-Kontorova model for bilayer graphene under stretch. 
a

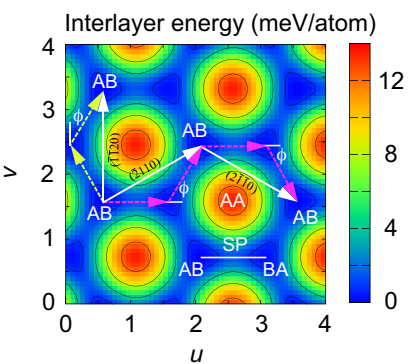

b

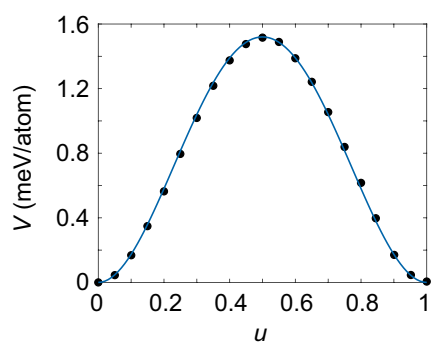

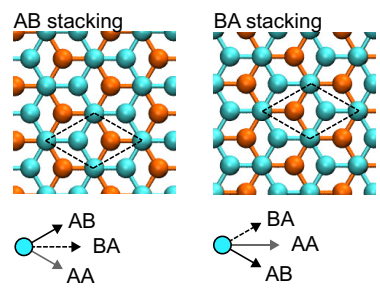

Figure S1 Potential energy surfaces (PES) and kink/antikink structures. a. Interlayer interaction energy in the graphene bilayer calculated as a function of their relative displacement along the armchair $(u)$ and zigzag $(v)$ directions. Calculations are performed using the registry-dependent Kolmogorov-Crespi $(\mathrm{K}-\mathrm{C})$ potential functions ${ }^{3}{ }^{3}$. The energy is plotted in unit of meV/atom, and the displacement in the unit of the $\mathrm{C}-\mathrm{C}$ bond length, $a=0.142 \mathrm{~nm}$. The interlayer distance is fixed at the value for a AB-stacking bilayer. The minimum energy path (MEP) connects AB and BA configurations through the saddle point (SP), as annotated by a white line. b. Interlayer interaction energy along the MEP, plotted as a function of $u$. The solid line is the approximation through a cosine function $V(u)=V_{\mathrm{SP}}\left[2 \cos \left(\frac{2 \pi}{3} u+\frac{2 \pi}{3}\right)+1\right]^{2}$, where the barrier is $V_{\mathrm{SP}}=1.52 \mathrm{meV} /$ atom. c. Lattice structures of bilayer graphene in $\mathrm{AB}$ and BA stacking orders. 

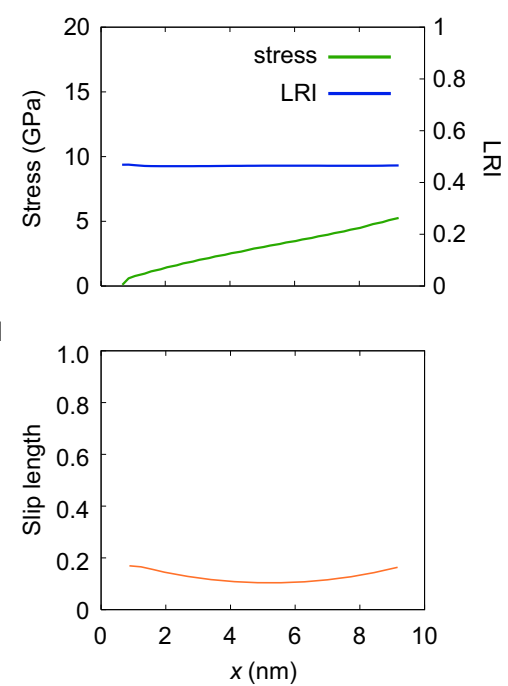

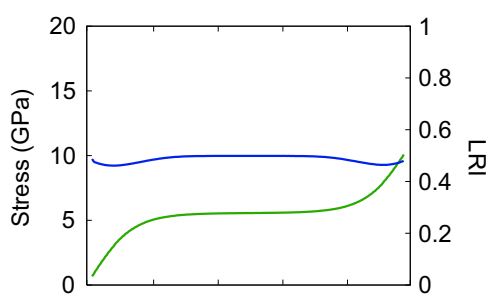

e

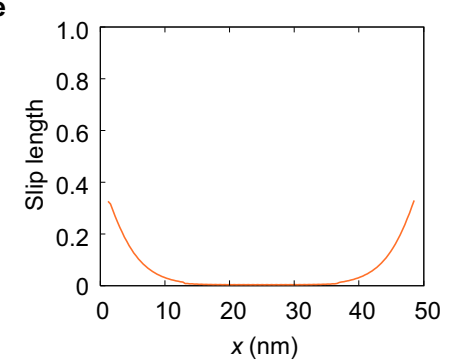

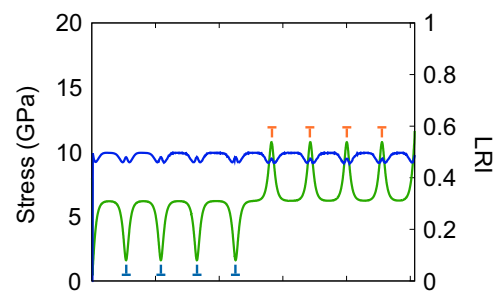

f

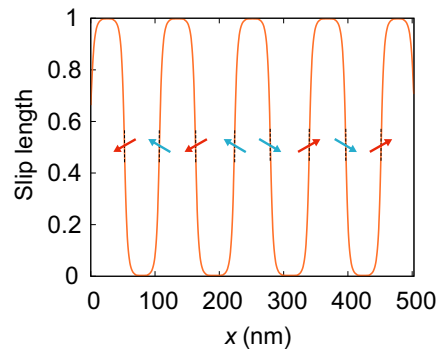

Figure S2 Stress and interlayer registry of bilayer graphene under stretch. Stress

distribution, local registry index (LRI, a-c) and slip length (SL, d-f) in the top layer of a graphene bilayer. The size of contact is $L=10,50$ and $500 \mathrm{~nm}$, respectively. The zigzag direction of graphene lattice aligns with the direction of stretch. 
a

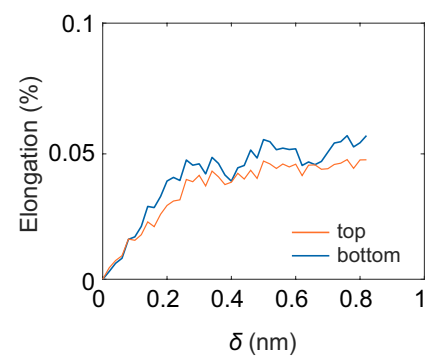

b

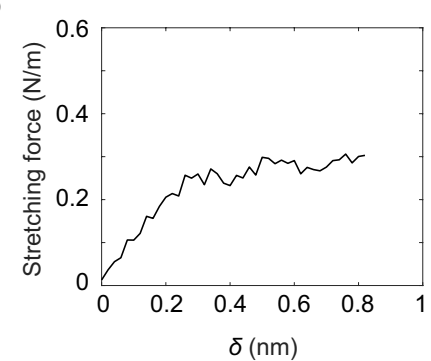

C

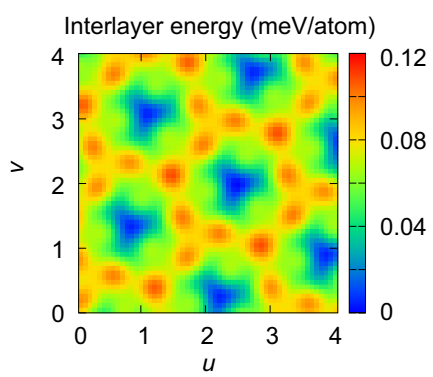

Figure S3 Mechanical responses and PES of bilayer graphene with a twisted angle.

Evolution of elongation (a) and stretching force (b) as functions of the end-to-end displacement $\delta$ for a graphene bilayer with $L=10 \mathrm{~nm}$. The armchair $(7,7)$ and chiral $(11,2)$ directions of the lattices align with the direction of stretch for the top and bottom layers, respectively. c. Interlayer interaction potential calculated for the bilayer with a corresponding twist angle of $8.2^{\circ}$, where the energy barriers is two orders of magnitude lower than that in a perfectly-aligned bilayer (Fig. $\mid$ S1). 


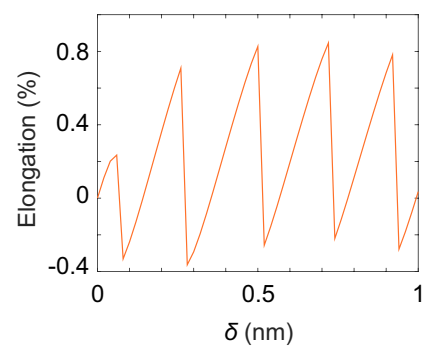

d

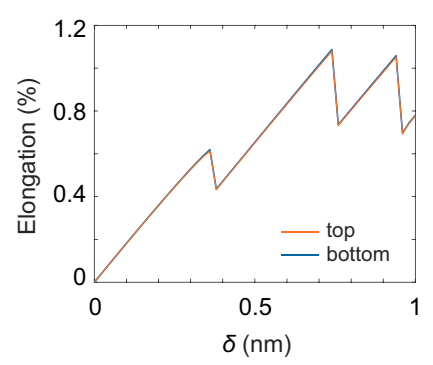

b

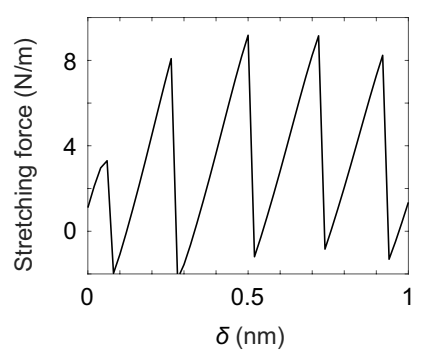

e

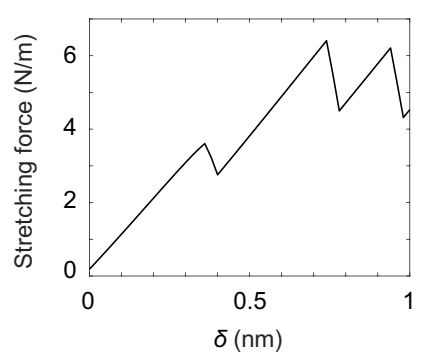

c

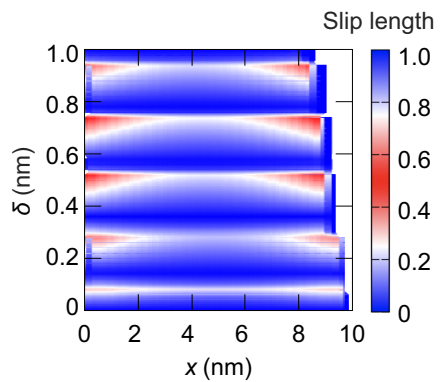

f

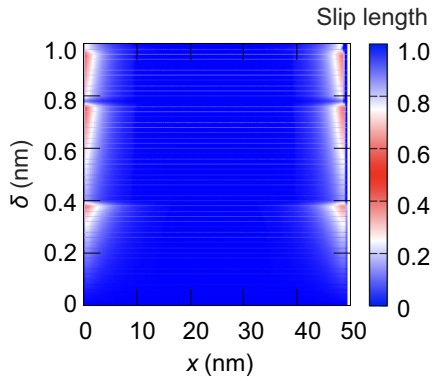

Figure S4 Mechanical responses and atomic slip in bilayer graphene under stretch.

Evolution of elongation $(\mathbf{a}, \mathbf{d})$, stretching force $(\mathbf{b}, \mathbf{e})$ and SL $(\mathbf{c}, \mathbf{f})$, calculated for graphene bilayers with $L=10$ (a-c) and $50 \mathrm{~nm}(\mathbf{d - f})$, respectively. Load is applied along the armchair direction. Stick-slip features are identified as the interface fails by sliding. As the size of contact $L$ increases, the load-dependent behaviors of stretching force and elongation change from oscillating patterns around 0 to load-bearing features. The SL data also show that overall sliding of the monolayers is suppressed as $L$ increases, which can be described using the deformable tensio-shear (DTS) model. 

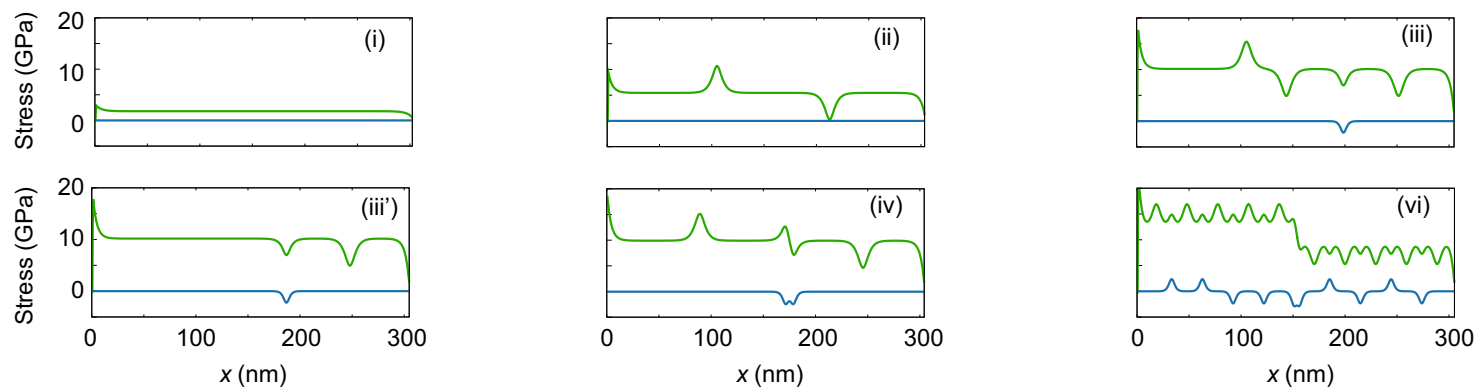

Figure S5 Stress distribution in the bottom layer for bilayer graphene. The overlap is

$L=300 \mathrm{~nm}$, and load is applied along the armchair direction. The results for the top layer are shown in Fig. 3d. 

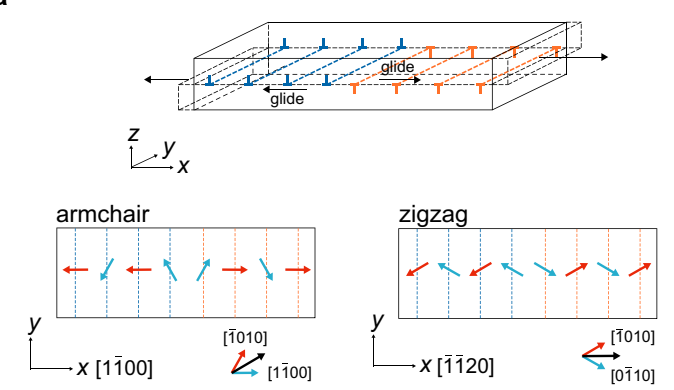

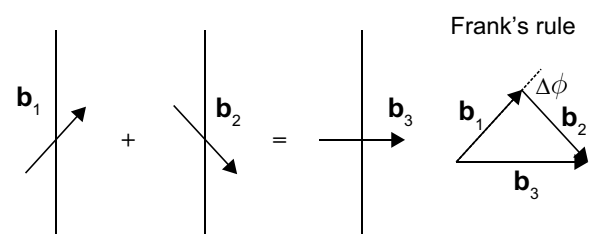

c

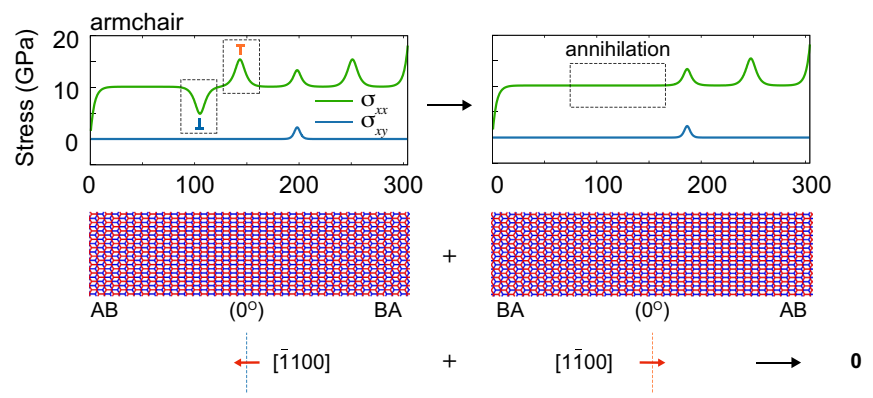

d

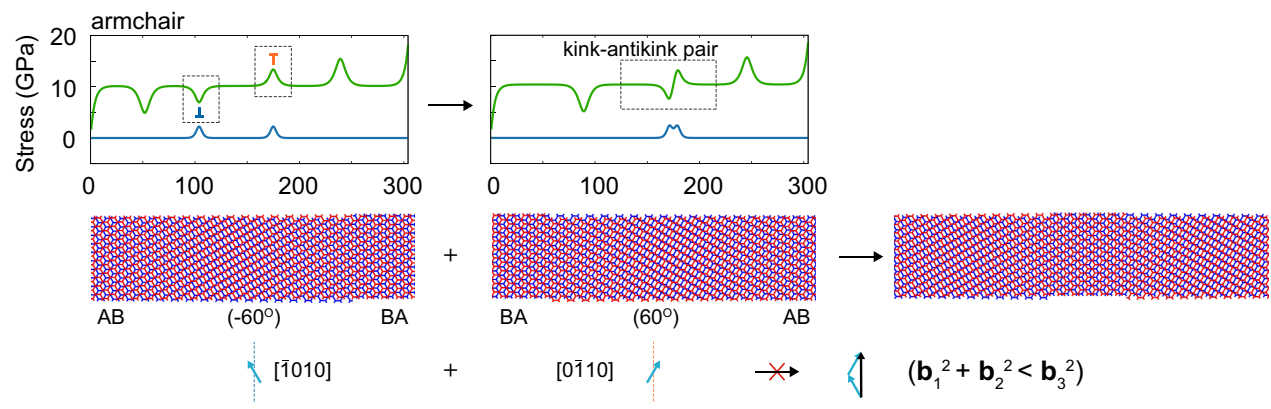

e
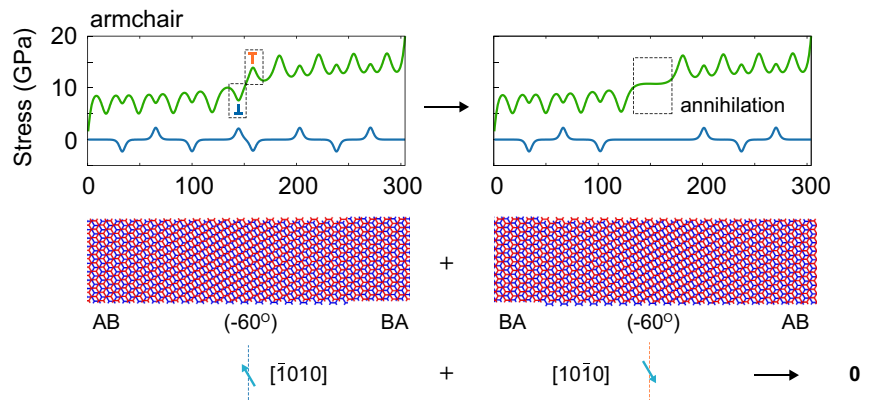

f
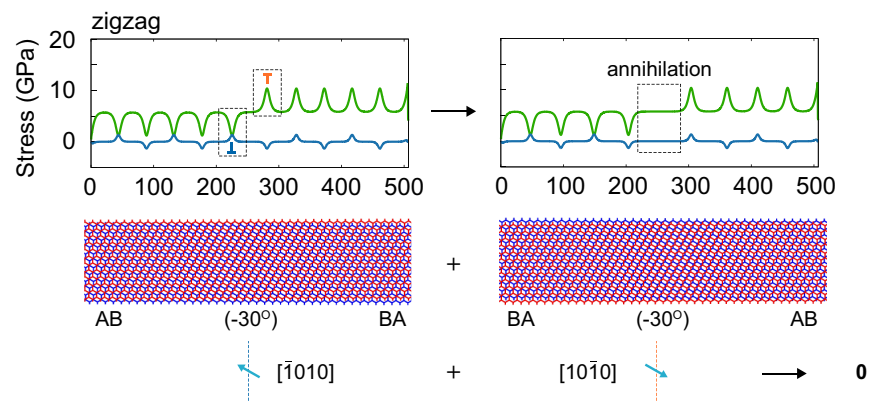
Figure S6 Evolution of kink and antikink structures. a. Illustration of dislocation gliding in bilayer graphene with stretch applied along the armchair or zigzag directions. The red and cyan arrows are the slip directions or Burgers vectors of dislocations. b. Frank's rule of dislocation reaction, which suggests that a reaction is feasible if $\mathbf{b}_{1}^{2}+\mathbf{b}_{2}^{2}>\mathbf{b}_{3}^{2}=\left(\mathbf{b}_{1}+\mathbf{b}_{2}\right)^{2}$. $\mathbf{c - f}$. Stress and dislocation reaction for stretch along the armchair (c-e, the Poisson's effect is included in panel e) or zigzag (f) directions, which determines the evolutionary dynamics of solitons in bilayer graphene. 
a

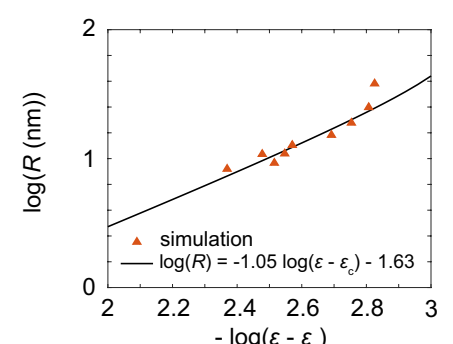

d

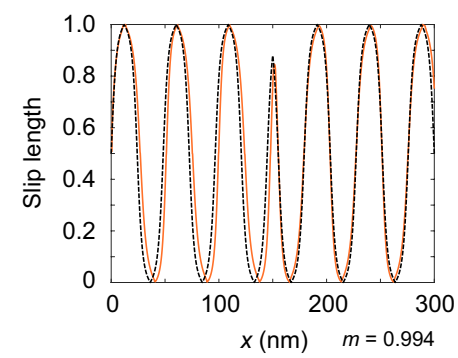

b

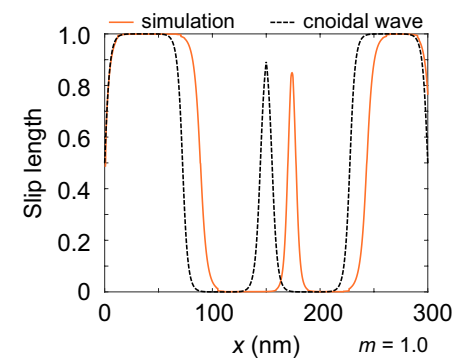

e

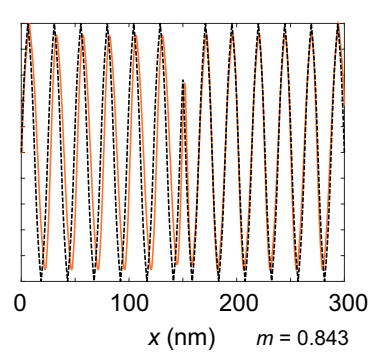

C

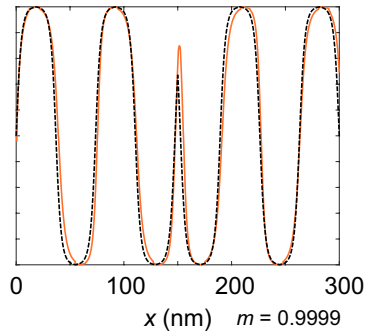

f

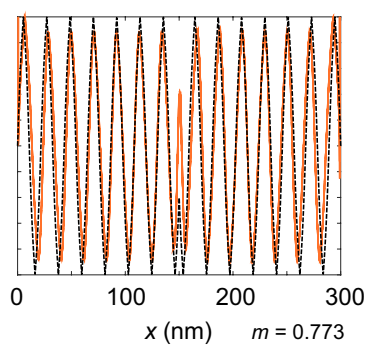

Figure S7 SL distribution in bilayer graphene. a. Scaling relation between $R$ and strain of the monolayers $\varepsilon, R \sim\left(\varepsilon-\varepsilon_{\mathrm{cr}}\right)^{-1.05}$, which is derived from the two-chain FK model and fitted through the simulation results (the coefficient of determination is $R^{2}=0.825$ ). Results are obtained from molecular dynamics (MD) simulations and the model approximation of Cnoidal waves (Eq. S14). The loading amplitude in panels $\mathbf{b}-\mathbf{f}$ is $\delta=3.74,4.0,4.02,5.02$ and $7.4 \mathrm{~nm}$, respectively. The bilayer with $L=300 \mathrm{~nm}$ is stretched along the armchair direction in the simulations. 
a

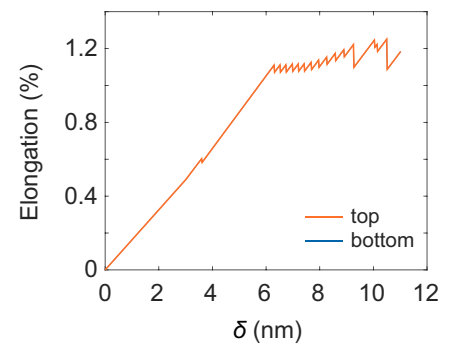

b

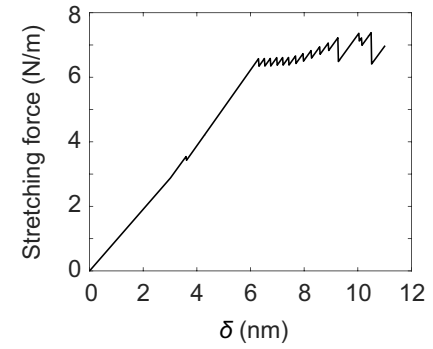

c

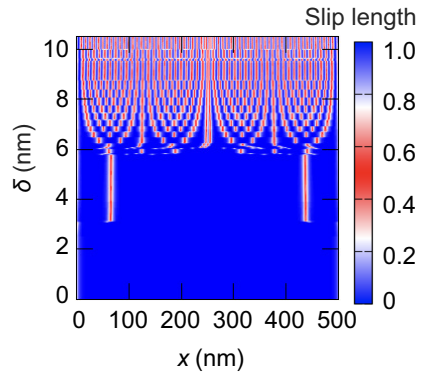

Figure S8 Mechanical responses and atomic slip of bilayer graphene stretched along the armchair direction. Evolution of elongation (a), stretching force (b), and SL (c) as functions of the end-to-end displacement $\delta$. The bilayer with $L=500 \mathrm{~nm}$ is stretched along the armchair direction. The thresholds for force and elongation to activate the formation of solitons are 6.6 $\mathrm{N} / \mathrm{m}$ and $1.1 \times 10^{-2}$. 
a

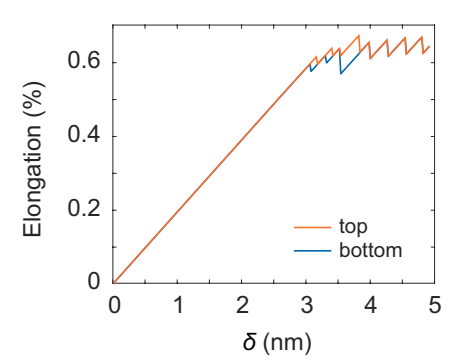

b

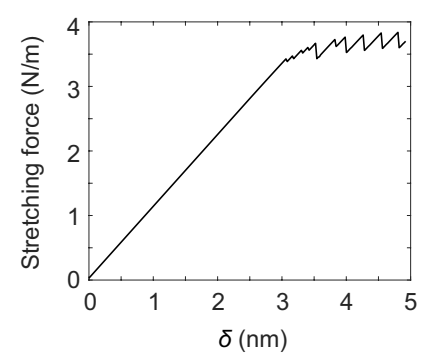

c

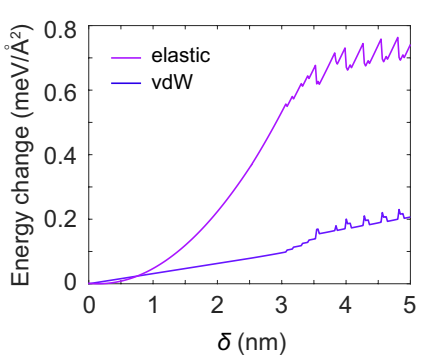

Figure S9 Mechanical responses of bilayer graphene stretched along the zigzag direction.

Evolution of elongation (a), stretching force (b), and changes in energy (c) as functions of the end-to-end displacement $\delta$. The bilayer with $L=500 \mathrm{~nm}$ is stretched along the zigzag direction. The thresholds of force and elongation to activate the formation of solitons are $3.42 \mathrm{~N} / \mathrm{m}$ and $6.2 \times 10^{-3}$, respectively. 
a

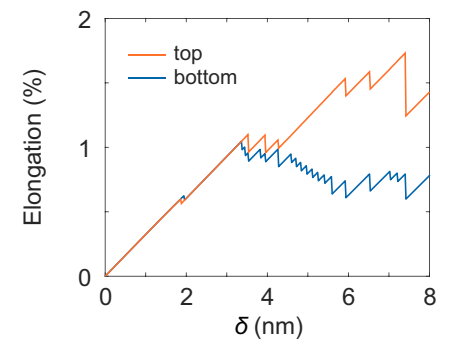

b

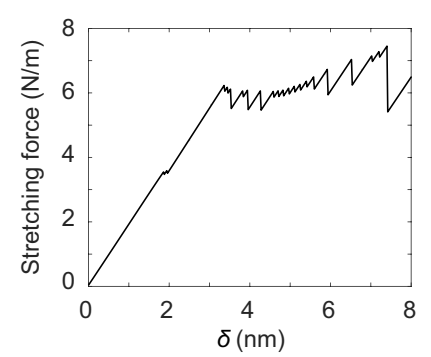

c

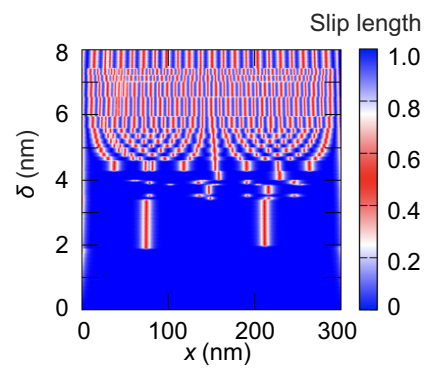

Figure S10 Mechanical responses and atomic slip of bilayer graphene with the bottom layer

constrained. Evolution of elongation (a), stretching force (b), and SL (c) as functions of the end-to-end displacement $\delta$. The bilayer with $L=300 \mathrm{~nm}$ is stretched along the armchair direction. The out-of-plane displacement of the bottom layer is restrained in the simulations. The symmetry between the two layers is thus broken. The thresholds of force and elongation to activate the formation of solitons are $6.2 \mathrm{~N} / \mathrm{m}$ and $1.1 \times 10^{-2}$, respectively. 


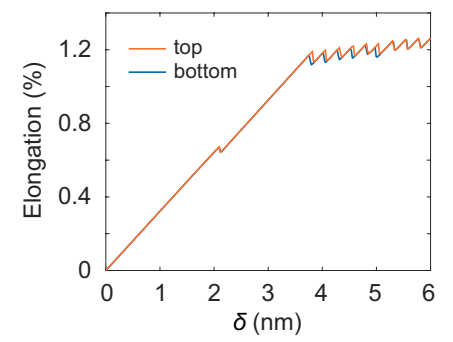

b

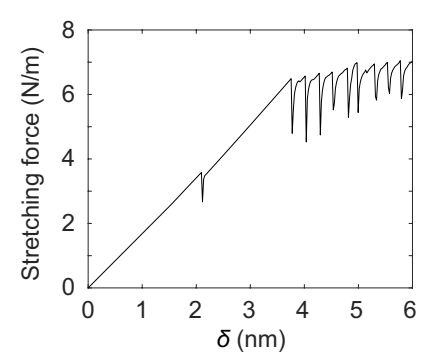

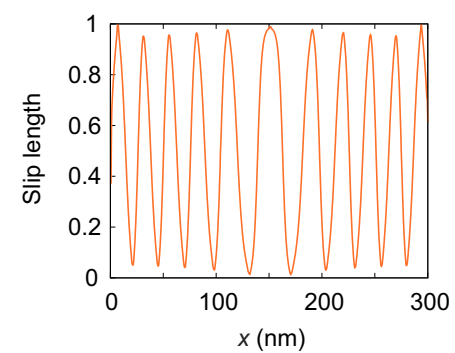

Figure S11 Mechanical responses and atomic slip of bilayer graphene with the Poisson's

effect. Evolution of elongation (a), stretching force (b) as functions of the end-to-end displacement $\delta$, and and SL (c) at $\delta=6 \mathrm{~nm}$. The bilayer with $L=300 \mathrm{~nm}$ is stretched along the armchair direction. Deformation in the transverse direction is allowed to include the Poisson effect. The thresholds of force and elongation to activate the formation of solitons are $6.67 \mathrm{~N} / \mathrm{m}$ and $1.2 \times 10^{-2}$, respectively. Similar evolutionary behaviors of soliton structures are identified as that in constrained simulations (Figs. 4). Solitons are created and drift to the position close to that in constrained simulations. 


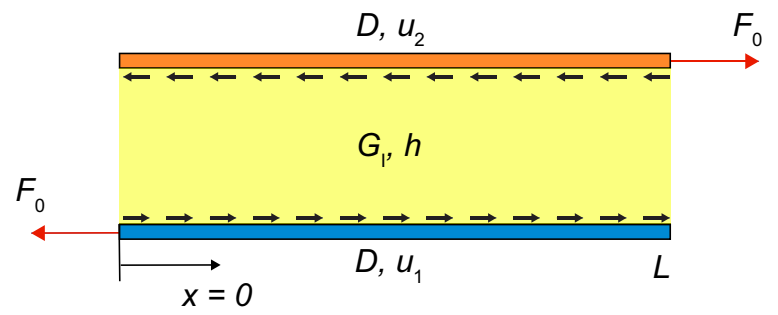

Figure S12 The deformable tensileshear (DTS) model of bilayer graphene. The graphene monolayers are considered as elastic sheets with tensile stiffness $D=Y t$, where $Y$ and $t$ are the Young's modulus and thickness. The interlayer interaction is modeled through a continuum with shear modulus $G_{\mathrm{I}}$. $h$ is the interlayer distance. 


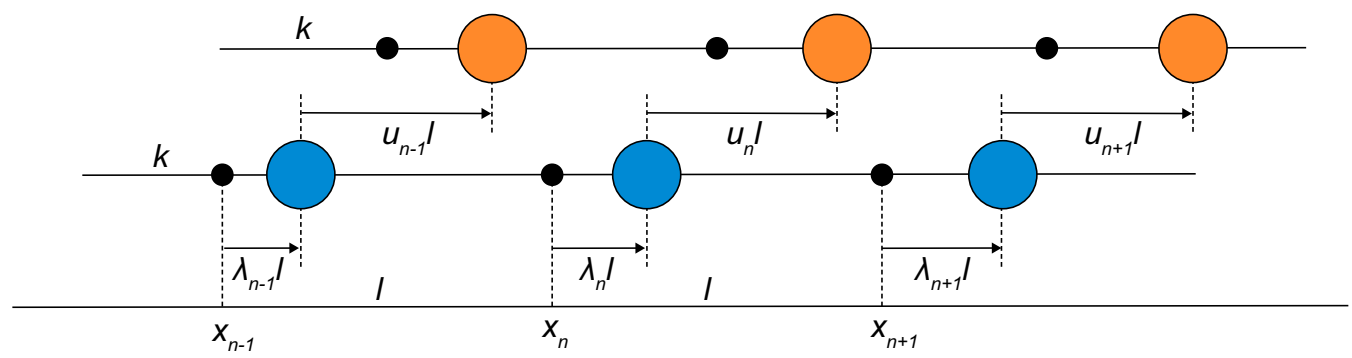

\section{Figure S13 The two-chain Frenkel-Kontorova model for bilayer graphene under stretch.}

The graphene sheets in a bilayer are modeled as chains of beads connected through elastic springs with a spring constant $k$. The original position of the bead is $x_{n}$, which is displaced by $\lambda_{n} l$ as the load is applied. $u_{n} l$ is the relative displacement between two beads in different layers. A $\mathcal{C}_{2}$ symmetry is assumed in the analysis following the simulation results. 


\section{References}

1. Plimpton, S. Fast parallel algorithms for short-range molecular dynamics. J. Comput. Phys. 117, 1-19 (1995).

2. Brenner, D. W. et al. A second-generation reactive empirical bond order (REBO) potential energy expression for hydrocarbons. J. Phys.: Condens. Matter 02, 783-802 (2002).

3. Kolmogorov, A. N. \& Crespi, V. H. Registry-dependent interlayer potential for graphitic systems. Phys. Rev. B 71, 235415 (2005).

4. Ouyang, W., Mandelli, D., Urbakh, M. \& Hod, O. Nanoserpents: Graphene nanoribbon motion on two-dimensional hexagonal materials. Nano Lett. 9, 6009-6016 (2018).

5. Naik, M. H., Maity, I., Maiti, P. K. \& Jain, M. Kolmogorovcrespi potential for multilayer transition-metal dichalcogenides: Capturing structural transformations in moiré superlattices. J. Phys. Chem. C 15, 9770-9778 (2019).

6. Zimmerman, J. A. et al. Calculation of stress in atomistic simulation. Model. Simul. Mater. Sci. Eng. 12, S319 (2004).

7. Liu, Y., Xie, B., Zhang, Z., Zheng, Q. \& Xu, Z. Mechanical properties of graphene papers. J. Mech. Phys. Solids 60, 591-605 (2012).

8. Hod, O. Quantifying the stacking registry matching in layered materials. Isr. J. Chem. 50, 506-514 (2010). 
9. Popov, A. M., Lebedeva, I. V., Knizhnik, A. A., Lozovik, Y. E. \& Potapkin, B. V. Commensurate-incommensurate phase transition in bilayer graphene. Phys. Rev. B 84, 045404 (2011).

10. Lebedeva, I. V. \& Popov, A. M. Commensurate-incommensurate phase transition and a network of domain walls in bilayer graphene with a biaxially stretched layer. Phys. Rev. B 99, 195448 (2019).

11. Hull, D. \& Bacon, D. J. Introduction to Dislocations (Elsevier, 2011).

12. Dai, S., Xiang, Y. \& Srolovitz, D. J. Structure and energetics of interlayer dislocations in bilayer graphene. Phys. Rev. B 93, 085410 (2016). 